Vol 11, Issue 8, 2018

\title{
INDIAN MEDICINAL PLANT TRIGONELLA FOENUM-GRAECUM: PHYTOCOMPOUNDS DETECTION AND PHARMACOLOGICAL STUDIES
}

\author{
AFAQUE AHMAD ${ }^{1}$, HIRDAY N VERMA ${ }^{1}$, KAPIL DEV ${ }^{2 *}$ \\ ${ }^{1}$ School of Life Sciences, Jaipur National University, Jaipur - 302 017, Rajasthan, India. ${ }^{2}$ Department of Biotechnology, Jamia Millia Islamia, \\ New Delhi - 110 025, Delhi, India. Email: kdev@jmi.ac.in \\ Received: 11 July 2017, Revised and Accepted: 30 April 2018
}

\begin{abstract}
Objective: Fenugreek (Trigonella foenum-graecum) is a medicinal plant of culinary origin commonly used as spice and vegetable. The present study was focused to identify the immunomodulatory applications of T. foenum-graecum.

Methods: The presence of phytochemicals and many biological activities such as antioxidant, antibacterial, and antifungal and anti-inflammatory activities were identified by various standard protocol respectively.

Results: The phytochemical analysis was flavonoids, alkaloids, phenols, etc., whereas no traces of carboxylic acid were found. Results showed that T. foenum-graecum aqueous and methanolic extract (TfgAE and TfgME) highly inhibited Escherichia coli (percentage mean growth inhibition [\% MGI] of $77.012 \pm 1.732 \%$ and $78.428 \pm 1.448 \%$ ) and least effective against Staphylococcus aureus (percentage MGI of $46.661 \pm 0.707 \%$ and $78.428 \pm 0.0076 \%$ ), respectively. TfgAE, TfgME, and ascorbic acid demonstrated highest percentage 2,2-diphenyl-1-picrylhydrazyl radical scavenging of $94.19316 \pm 0.0047 \%$, $94.7942 \pm 0.0050 \%$, and $99.67775 \pm 0.0053 \%$ and lowest of $17.18339 \pm 0.0059 \%, 24.47114 \pm 0.0052 \%$, and $61.81149 \pm 0.0046 \%$, respectively. Ferric reducing antioxidant power assay of TfgAE and TfgME showed maximum of $1.0527 \pm 0.065 \mathrm{mM}$ and $1.0067 \pm 0.041 \mathrm{mM}$ and minimum value of $0.3027 \pm 0.052 \mathrm{mM}$ and $0.275 \pm 0.057 \mathrm{mM}$, respectively, as compared to the ascorbic acid $(1.1847 \pm 0.049 \mathrm{mM}$ and minimal at $0.373 \pm 0.032 \mathrm{mM})$. TfgAE and TfgME showed anti-inflammatory activity with the highest inhibition of albumin denaturation of $44.93771 \pm 0.0071 \%$ and $39.14707 \pm 0.0068 \%$ and the lowest of $21.32969 \pm 0.0075 \%$ and $15.53905 \pm 0.0065 \%$, respectively. The other assay revealed that TfgAE and TfgME demonstrated the highest proteinase inhibition of $29.94818 \pm 0.0071 \%$ and $74.337 \pm 0.0073 \%$ and the lowest of $14.47679 \pm 0.0082 \%$ and $25.327 \pm 0.0079 \%$, respectively, at extract volume or amount of $1000 \mu \mathrm{l}$ and $100 \mu \mathrm{l}$.
\end{abstract}

Conclusion: Results showed T. foenum-graecum might act as strong natural antibiotic agent and antioxidant agent and can be used against inflammatory diseases.

Keywords: Food chemistry, Phytocompounds, Antibacterial, Antioxidant, Anti-inflammatory, Fenugreek, Trigonella foenum-graecum.

(C) 2018 The Authors. Published by Innovare Academic Sciences Pvt Ltd. This is an open access article under the CC BY license (http://creativecommons. org/licenses/by/4. 0/) DOI: http://dx.doi.org/10.22159/ajpcr.2018.v11i8.21200

\section{INTRODUCTION}

Food is just a major concern of human health that controls the metabolism and functioning of the human body. The food ingredients, namely proteins, fat, carbohydrates, vitamins, antioxidants, fibers, and minerals prevent the various chronic diseases and metabolic disorders [1]. Plant-based natural antioxidants are drawing attention of the researchers, industrialist, and users toward them because they cure the body of various diseases such as cancer, atherosclerotic heart disorders, and other epidemics [2]. The secondary metabolites found in the herbal plants possess various biofunctional compounds which could be used as drugs, food additives, colorants, flavorants, and as pesticide agents [3].

Fenugreek (Trigonella foenum-graecum L.) being the ancient medicinal herbs that belong to the family Fabaceae possesses the highest medicinally important bioactive compounds. There is an abundant dietary fiber in fenugreek seeds which help in the downregulation of blood sugar levels in diabetic patients. Fenugreek (T. foenum-graecum) plant as a whole or its plant parts are used to prepare the powdered form and that can be used for solvent extraction and can prepare various types of extracts that possess bioactive compounds of medicinal use. There are high toxic oils, and other bioactive ingredients in the fenugreek seed include volatile oils and alkaloids show toxic effects on bacteria, parasites, and fungi [4]. In modern Egypt, it may be used as wheat and maize flour to prepare bread [5]. Fenugreek might be used as lactation stimulant and flavorant seeds in India [6]. It might be exploited in labor pain and delivery in the old Rome while they might be used as a tonic for the treatment of edema and legs weakness in the China [7]. Other uses of fenugreek seeds include anti-diabetic property $[8,9]$.

Fenugreek plant is a reservoir of several ethnomedicinal properties that include antimicrobial, laxative, uterine tonic, expectoral, restorative, galactagogue, anti-carcinogenic, anti-inflammatory, anticholesterolemic, antiviral, and antioxidant [39,10]. This plant also possesses several other potentials to fight against fever, body pain and fat, swelling, and might induce lactation and sex hormones. Fenugreek compounds are biofunctional in nature that could act as a safeguard against various diseases such as malaria, cancer, bacterial, and viral diseases [11, 3]. Fenugreek possesses abundant of polyphenolics compounds that are known to prevent peroxidation and hence decreases the oxidative hemolysis of erythrocytes [12,13]. Moreover, their normal consumption may reduce triglycerides and cholesterol concentrations in the blood [14], prevent cancer [15], and regulate diabetes mellitus [16].

This culinary herb is being used in pharmacology and is used for the treatment of diseases. It may also be used as a dietary antioxidant because it might reduce the hydrogen peroxide-induced peroxidation in the mitochondria of liver and provide protection to the cellular organelles from oxidative stress [17]. These days, fenugreek is used in pharmacology as a medicinal plant and might provide treatments for the fatal diseases. There were some reports available (to the best of my knowledge) on the seeds of fenugreek which were tested against various human pathogenic 
bacterial strains. In the recent study, we have evaluated the antimicrobial activity of fenugreek against pathogenic bacteria. This study revealed that methanolic seed extracts are having strong antibacterial activities against some bacterial pathogens. The present study is however intended toward the identification of the phytochemical ingredients found in the extracts followed by the comparative appraisal of in vitro antibacterial, antioxidant, and anti-inflammatory activities of the aqueous and methanolic extract of T. foenum-graecum.

\section{METHODS}

\section{Sample collection}

The seed samples of fenugreek ( $T$. foenum-graecum) were obtained from the local ayurvedic clinic.

\section{Chemicals and reagents}

Various chemicals used for antioxidant assays 2,2-diphenyl-1picrylhydrazyl (DPPH), and 2,4,6-Tris(2-pyridyl)-1,3,5-triazine (TPTZ) and trypsin were purchased from Sigma-Aldrich, USA. Other chemicals (analytical grade) which were used for antibacterial, antifungal, and anti-inflammatory assay were obtained from SRL, Himedia, and Merck.

\section{Test organisms}

The tested bacterial strains were obtained from NCCS, Pune, India. The bacterial cultures were then maintained at $4{ }^{\circ} \mathrm{C}$ on slants of nutrient agar.

\section{Preparations of aqueous and methanolic extract of T.foenum-graecum seeds}

The extracts from T. foenum-graecum were prepared by dissolving powdered Trigonella in distilled water (DW) and methanol to obtain aqueous and methanolic extracts, respectively. The plant sample was soaked for 48-72 $\mathrm{h}$ in the solvents for the extraction of almost all valuable phytocompounds. Further, the samples were centrifuged at $6000 \mathrm{rpm}$ for $15 \mathrm{~min}$ for the settling down of debris at the bottom in the form of a pellet, and the supernatants were collected. The supernatant was named as T. foenum-graecum aqueous extract (TfgAE) and T. foenumgraecum methanolic extract (TfgME) and again filtered through a membrane filter $(0.25 \mu \mathrm{m})$ and used for the further bioactivities.

\section{Identification of phytocompounds from TfgAE and TfgME}

The extracts TfgAE and TfgME were screened qualitatively to determine the presence of phytochemicals including amino acids, flavonoids, tannins, quinones, alkaloids, phenols, carbohydrates, glycosides, steroids, carboxylic acids, resins, proteins, saponins, and terpenoids according to the method described by Harborne with some minor modifications [18].

\section{Evaluation of antimicrobial activity \\ Determination of antibacterial activity by broth microdilution assay}

Antibacterial activity of the TfgAE and TfgME was evaluated against various Gram-positive and Gram-negative strains (Bacillus subtilis [MTCC 736], Pseudomonas aeruginosa [MTCC 2453], Staphylococcus aureus [MTCC 902], and Escherichia coli [MTCC 443]) as described by Barbade and Datar with some modifications [19]. The bacterial primary cultures were prepared by inoculating bacterial colony from agar plate into the Luria broth (LB) media followed by incubation at $37^{\circ} \mathrm{C}$ for $12 \mathrm{~h}$. The fresh LB media were used to dilute the overnight cultures, and then TfgAE and TfgME extracts were added and incubated at $37^{\circ} \mathrm{C}$ for $12-14 \mathrm{hrs}$. Results were compared to the positive control culture (LB media and bacterial inoculums) and negative control (media+bacteria+ampicillin). The experiment was repeated thrice for the confirmation. The absorbance was recorded at $600 \mathrm{~nm}$. Using the given formula, the percentage inhibition was calculated:

Percentage mean growth inhibition $(\% \mathrm{MGI})=[(\mathrm{Ac}-\mathrm{At}) / \mathrm{Ac}] \times 100$,

Ac and At here represent the absorbance of control and test sample, respectively.

\section{Evaluation of antioxidant potential of TfgAE and TfgME DPPH assay}

TfgAE, TfgME, and the ascorbic acid were checked for their antioxidant potential due to the basic effect of free radical scavenging of the stable DPPH as per the method of Goveas and Abraham with minor modifications [20]. TfgAE and TfgME extracts were serially diluted in DW and methanol, respectively. DPPH $(0.1 \mathrm{mM})$ was prepared in $80 \%$ methanol and mixed with the serially diluted sample and standard ascorbic acid solutions separately. The reaction mixtures were placed in the dark for about 5-10 min and the absorbance had been recorded at $517 \mathrm{~nm}$ spectrophotometrically. DPPH solution was used as a control. The sample was taken as their respective blank. Using the formula given below the DPPH scavenging was calculated as:

DPPH scavenging Activity $(\%)=[(\mathrm{Ac}-\mathrm{At}) / \mathrm{Ac}] \times 100$,

Here Ac and At represent the absorbance of control and test sample, respectively.

\section{Ferric reducing antioxidant power (FRAP) assay}

This FRAP assay to determine the antioxidant potential of TfgAE and TfgME was assessed using the method of Sudha et al. with few modifications [21]. Serially diluted TfgAE and TfgME were added to the freshly prepared FRAP reagent and the incubation of reaction mixture for 4-10 min in the dark. The formation of colored complex, i.e., ferrous tripyridyltriazine complex increased the absorbance which was recorded at $593 \mathrm{~nm}$. The FRAP reagent was used as blank in the cuvette. The absorbance of each diluted extract and standard was expressed in $\mathrm{mM}$ of FRAP value.

\section{Superoxide dismutase (SOD) assay}

SOD assay was performed using the protocol as described by Kakkar et al. with few modifications [22]. The extracts, i.e., TfgAE and TfgME were mixed with Phenazine methosulfate (PMS), sodium pyrophosphate buffer, and nitro blue tetrazolium chloride (NBT). Further, the addition of nicotinamide adenine dinucleotide (NADH) to the above mixture, the whole reaction was initiated and then incubated at $30^{\circ} \mathrm{C}$ for $10-15 \mathrm{~min}$. The glacial acetic acid was used to terminate the reaction by adding it to the assay mixture. n-butanol was also added to the assay mixture, and the intensity at $560 \mathrm{~nm}$ of the chromogen developed in a layer of butanol was recorded.

\section{Reduced glutathione (GSH) assay}

Reduced GSH was determined according to the method as described by Moron et al. with some modification [23]. TfgAE and TfgME of different concentration were mixed with $0.2 \mathrm{M}$ sodium phosphate buffer ( $\mathrm{pH}$ 8.0). Standard GSH was also prepared with concentrations of 2-10 nm range. Further, 5,5'-Dithiobis 2-nitrobenzoic acid (DTNB) freshly prepared solution was mixed to the reaction mixture, and then the solution was incubated for $10 \mathrm{~min}$. The intensity of the yellow color developed was recorded at $412 \mathrm{~nm}$. The results were expressed in $\mu \mathrm{M} / \mathrm{min} / \mu \mathrm{l}$ of both the extracts.

\section{Catalase assay}

Catalase activity was assayed as described by Jambunathan et al. with certain modifications [24]. $\mathrm{H}_{2} \mathrm{O}_{2}$ and phosphate buffer were taken in a cuvette, and the few amount of TfgAE and TfgME extracts was added continuously and then mixed thoroughly. The $\mathrm{H}_{2} \mathrm{O}_{2}$ degradation by the extracts was recorded as a drop in the absorbance by 0.05 units and noted to be at $240 \mathrm{~nm}$. The $\mathrm{H}_{2} \mathrm{O}_{2}$ - Phosphate buffer was used as control in this assay. Results were expressed by the amount of enzyme present in the extracts which decreased the absorbance at $240 \mathrm{~nm}$ by $0.05-0.06$ units and termed as one enzyme unit. The ultraviolet (UV) absorption of $\mathrm{H}_{2} \mathrm{O}_{2}$ recorded at $240 \mathrm{~nm}$ was due the degradation by the enzyme catalase. 
Assessment of anti-inflammatory activity

\section{Albumin denaturation inhibition}

The anti-inflammatory assay was performed by the method of Mizushima et al. with some changes [25]. The TfgAE and TfgME were serially diluted and then mixed with $1 \%$ aqueous solution of bovine serum albumin (BSA) fraction. The assay mixtures were incubated at $37^{\circ} \mathrm{C}$ for $30 \mathrm{~min}$ and then heat was given at $60^{\circ} \mathrm{C}$ for $15 \mathrm{~min}$. BSA was used as control and water was taken as blank. Aspirin $(100 \mu \mathrm{g} / \mathrm{ml})$ was used as standard anti-inflammatory drug. When the samples were cooled down, the turbidity was recorded at $660 \mathrm{~nm}$. Using the below formula percentage inhibition of albumin denaturation was calculated as:

Percentage of inhibition $=\left(\mathrm{Abs}_{\mathrm{C}}-\mathrm{Abs}_{\mathrm{T}}\right) \times 100 / \mathrm{Abs}_{\mathrm{C}}$

$\mathrm{Abs}_{\mathrm{C}}$ and $\mathrm{Abs}_{\mathrm{T}}$ were the absorbance of control and test sample, respectively.

\section{Proteinase inhibitory action}

The bioactivity was done by Oyedepo and Femurewa with some minor modifications [26]. The reaction mixture contained $20 \mathrm{mM}$ Tris- $\mathrm{HCl}$ buffer ( $\mathrm{pH}$ 7.4) and serially diluted extracts, i.e., TfgAE and TfgME. Further, approximately $0.05 \mathrm{mg}$ trypsin was added to it. At $37^{\circ} \mathrm{C}$, the sample was incubated for 15-25 min, and then some amount of $1 \%$ casein was added followed by the additional incubation for $25 \mathrm{~min}$. Perchloric acid was added to the sample mixture to arrest the reaction. The absorbance of the supernatant was read at $210 \mathrm{~nm}$. The percentage of proteinase inhibitory activity was calculated by the given formula.

$\%$ proteinase inhibition $=\left(A_{\text {control }}-A_{\text {sample }}\right) \times 100 / A_{\text {control. }}$

Where $A_{\text {control }}$ and $A_{\text {sample }}$ are the absorbance at $210 \mathrm{~nm}$ for control and sample respectively.

\section{Determination of antifungal activity}

TfgAE and TfgME extracts were tested for their antifungal activity against the Candida cells (ATCC 10261 and ATCC 90028) by assessing the minimum inhibitory concentration using the broth microdilution method [27]. Using the extracts or without any extract in the media, the fungal cultures were grown. First, the 2 -fold dilutions of the extracts were carried out. Then, by the disc diffusion method, the antifungal activity of TfgAE and TfgME was determined in the solid YEPD agar plate. The fungal cells, i.e., Candida strains $\left(10^{5}\right.$ cells $\left./ \mathrm{ml}\right)$ were inoculated in the YEPD agar (molten $\sim 40^{\circ} \mathrm{C}$ ) and then poured into a Petri plates. Further, the discs were kept on the solid agar plates and different concentrations of TfgAE and TfgME extracts were poured on the disc in $10 \mu \mathrm{l}$ volume. The plates were then incubated in an incubator for $36-73 \mathrm{~h}$. The zone of inhibition was recorded with its average diameter in $\mathrm{mm}$.

\section{Statistical analysis}

The experiments were done in triplicates. Results were expressed as graphs representing mean \pm standard error of mean using the software GraphPad Prism 5.

\section{RESULTS}

Identification of phytocompounds from TfgAE and TfgME

Qualitative phytochemical analysis of aqueous and methanolic extracts of T. foenum-graecum plant revealed the presence of phenolics and non-phenolics phytocompounds such as total phenols, tannins, and flavonoids, alkaloids, sterol, resins, terpenoids, xanthoproteins, quinines, glycosides, and saponins while the steroids, tannins, and carboxylic acids were absent in both types of extracts. Other compounds were found in fairly detectable quantity (Table 1 ).

\section{Assessment of antimicrobial activity}

Antibacterial activity

Antibacterial potential of the TfgAE and TfgME extracts was determined against various bacterial strains by evaluating the percentage MGI in the presence of these extracts. Results were compared to the control sample where only LB media and bacterial inoculum were added. The results obtained suggested that TfgAE and TfgME possessed the bactericidal property and showed inhibition of growth of microorganisms in their presence. It was found that TfgAE was most effective against $E$. coli (ATCC 25922) with percentage MGI of $77.012 \pm 1.732 \%$ and was least effective against $S$. aureus with percentage MGI of $46.661 \pm 0.707 \%$. TfgME showed maximum percentage MGI of $78.428 \pm 1.448 \%$ against $E$. coli whereas least against S. aureus, i.e. percentage MGI of $78.428 \pm 0.0076 \%$. The standard antibiotic ampicillin showed almost complete inhibition against all the bacterial strains at the final concentration of $0.5 \mathrm{mg} / \mathrm{ml}$ (Fig. 1).

\section{Evaluation of Antioxidant Potential of TfgAE and TfgME} DPPH Assay

T. foenum-graecum showed scavenging of DPPH free radical in a dosedependent manner which was shown in Fig. 2. The DPPH radical scavenging activity by TfgAE and TfgME was shown with the highest and lowest scavenging at amount $500 \mu \mathrm{l}$ and $50 \mu \mathrm{l}$ of the extracts. TfgAE demonstrated the highest percentage DPPH radical scavenging of $94.19316 \pm 0.0047 \%$ and the lowest of $17.18339 \pm 0.0059 \%$ while the TfgME showed the highest \% DPPH radical scavenging of $94.7942 \pm 0.0050 \%$ and the lowest of $24.47114 \pm 0.0052 \%$. The results

Table 1: Tabular representation of the presence of phytochemicals extracted from the $\boldsymbol{T}$. foenum-graecum methanolic and aqueous extract

\begin{tabular}{lll}
\hline Phytochemical constituents & TfgAE & TfgME \\
\hline Alkaloids & + & ++ \\
Flavonoids & + & ++ \\
Steroids & + & ++ \\
Saponins & + & ++ \\
Phenols & + & ++ \\
Resins & + & ++ \\
Tannins & ++ & + \\
Terpenoids & + & ++ \\
Xanthoproteins & ++ & + \\
Quinones & + & ++ \\
Glycosides & + & ++ \\
Carboxylic acid & - & - \\
\hline
\end{tabular}

T. foenum-graecum: Trigonella foenum-graecum, TfgAE: Trigonella foenum-graecum aqueous extract, TfgME: Trigonella foenum-graecum methanolic extract

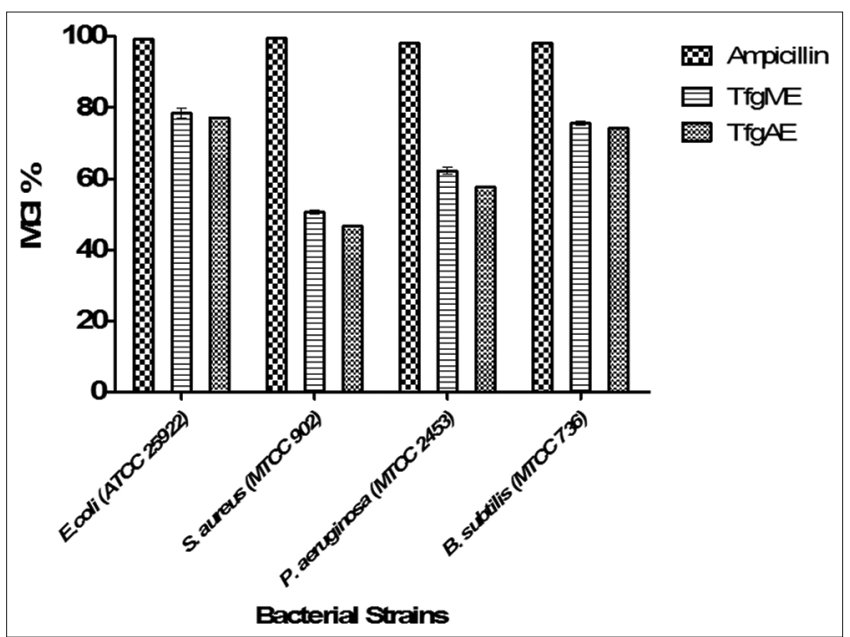

Fig. 1: Antibacterial potential of a methanolic and aqueous extract of Trigonella foenum-graecum was calculated, and the results were expressed in percentage mean growth inhibition. Experiment was performed in triplicates. Results were expressed in the form of means \pm standard error of mean 
were quite comparable to that of standard, i.e., ascorbic acid with maximum percentage DPPH scavenging of $99.67775 \pm 0.0053 \%$ and minimum of $61.81149 \pm 0.0046 \%$.

\section{FRAP assay}

The results showed that FRAP value of T. foenum-graecum increases in according to the concentration-dependent manner. The FRAP assay showed the highest absorbance at $500 \mu \mathrm{l}$ for both TfgAE and TfgME extracts, and the lowest was recorded at $50 \mu \mathrm{l}$ with a maximum FRAP value $1.0527 \pm 0.065 \mathrm{mM}$ and $1.0067 \pm 0.041 \mathrm{mM}$ and a minimum value of $0.3027 \pm 0.052 \mathrm{mM}$ and $0.275 \pm 0.057 \mathrm{mM}$, respectively, as compared to standard maxima at $1.1847 \pm 0.049 \mathrm{mM}$ and minimal at $0.373 \pm 0.032$ $\mathrm{mM}$, respectively. These extracts reacted with ferric tripyridyltriazine (Fe'IIITTZ) complex which produced a blue colored ferrous tripyridyltriazine (Fe ${ }^{\mathrm{II}}$-TPTZ) complex. It had been cleared that T. foenumgraecum showed fair antioxidant activity which was comparable to ascorbic acid, a standard antioxidant (Fig. 3) from the given observations.

\section{SOD assay}

SOD assay is dependent on inhibition of formation of NADH-PMSnitroblue tetrazolium formazon. The conversion of superoxide free radicals to hydrogen peroxide and molecular oxygen is due to SOD assay. It is a major protection for aerobic cells in resisting the toxic effects of superoxide radicals. They represent the main enzymatic source of peroxides and belong to a large family of isoenzymes that relate cellular response to the oxidative stress. The amount of extract which may inhibit NBT reduction up to $50 \%$ in 1 min termed as one unit of enzyme activity. The degradation of superoxide radicals per minute was evaluated, and this radical was degraded on an average unit of 0.006 by TfgAE and 0.023 units by TfgME (Fig. 4).

\section{Reduced GSH assay}

GSH peroxidase catalyzes the reduction of hydrogen peroxides and functions to protect the cell from oxidative damage. GSH is the necessary part of non-enzymatic antioxidants, and it can reduce peroxynitrite with the formation of oxidized glutathione, which results in the formation of GSH by NADPH-dependent GSH reductase. DTNB was used as a control, and the DTNB with different concentration of TfgAE and TfgME was used as a test sample. The concentration of GSH was expressed in $\mu \mathrm{M} / \mathrm{min} / \mu \mathrm{l}$ extract and estimated up to $200 \mu \mathrm{l}$ of both extracts. GSH was found in low concentration at $50 \mu \mathrm{l}$ and in high concentration at $200 \mu \mathrm{l}$ volume of both TfgAE and TfgME (Fig. 5).

\section{Catalase assay}

The results revealed that the absorbance of UV light by hydrogen peroxide decreases when scavenged or deteriorated by the enzyme catalase found in the extracts which can be measured at $240 \mathrm{~nm}$. When the absorbance reduced, the enzyme activity can though be portrayed as the concentration of hydrogen peroxide is decreasing significantly in a dose-dependent manner. The $100 \%$ hydrogen peroxide was degraded to $75.16246 \pm 0.0063 \%$ and $71.58474 \pm 0.0067 \%$ when the initially small amount of TfgAE and TfgME was added to the cuvette, respectively. Degradation per minute observed up to $10 \mathrm{~min}$, and finally, there was approximately $7.889728 \pm 0.0068 \%$ and $2.229903 \pm 0.0059 \%$ radical was remained (Fig. 6).

\section{Evaluation of anti-inflammatory activity}

\section{Albumin denaturation inhibition}

Infection or damage in the body tissue shows response against this infection through inflammation. Protein denaturation is said to be one of the major causes of inflammation. Due to this, we have tried to find out the ability of T. foenum-graecum to inhibit protein denaturation. The results showed that TfgAE and TfgME were responsible for inhibiting thermally induced albumin denaturation at different concentrations. The TfgAE showed the highest percentage inhibition of albumin denaturation of $44.93771 \pm 0.0071 \%$ and the lowest of $21.32969 \pm 0.0075 \%$ while TfgME resulted in maximum percentage

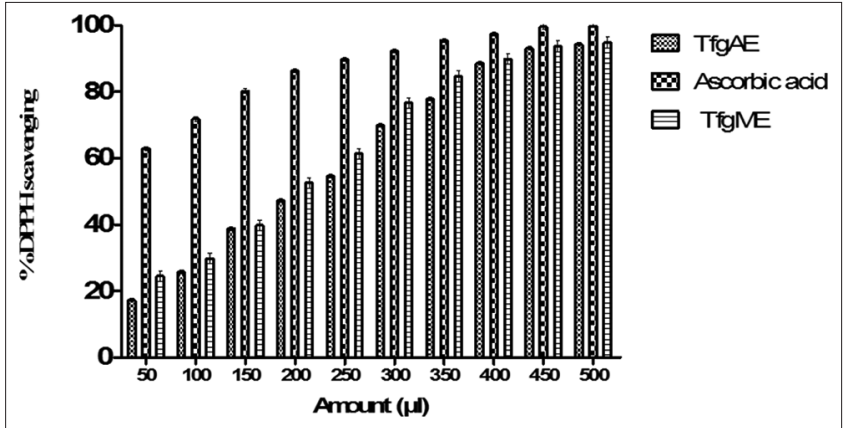

Fig. 2: 1, 1-diphenyl-2-picrylhydrazyl free radical scavenging activity of methanolic and aqueous extract were calculated and compared to ascorbic acid, i.e., standard. The activity increased in time- and concentration-dependent manner. Experiment was performed in triplicates. Results were expressed in the form of means \pm standard error of mean.

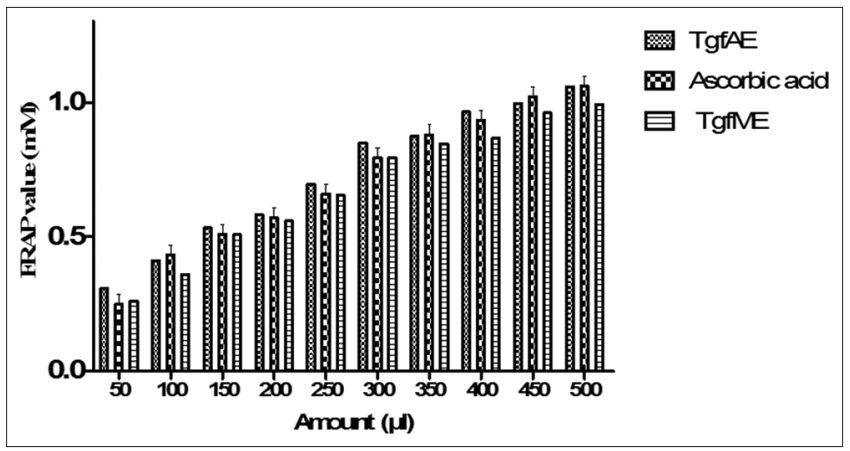

Fig. 3: Ferric reducing the antioxidant power of methanolic and aqueous extract of Trigonella foenum-graecum was estimated and compared to ascorbic acid as standard. The results were expressed in mM. Experiment was performed in triplicates. Results were expressed in the form of means \pm standard error of mean.

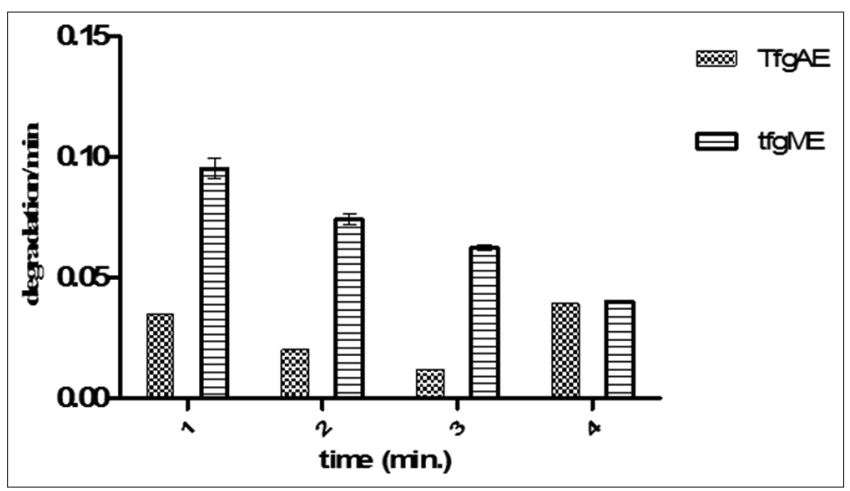

Fig. 4: Superoxide radical level significantly decreased with increase in the concentration of methanolic and aqueous extract. The results were expressed in degradation/min. Experiment was performed in triplicates. Results were expressed in the form of means \pm standard error of mean.

inhibition of $39.14707 \pm 0.0068 \%$ and minimum percentage inhibition of $15.53905 \pm 0.0065 \%$ at extract volume or amount of $1000 \mu \mathrm{l}$ and $100 \mu$ l, respectively (Fig. 7).

\section{Proteinase inhibitory activity}

Results exhibited significant anti-proteinase activity at different concentrations of TfgAE and TfgME. The TfgAE demonstrated the 
highest percentage proteinase inhibition of $29.94818 \pm 0.0071 \%$ and the lowest of $14.47679 \pm 0.0082 \%$ while TfgME resulted in maximum proteinase inhibition of $74.337 \pm 0.0073 \%$ and minimum inhibition of $25.327 \pm 0.0079 \%$ at extract volume or amount of $1000 \mu \mathrm{l}$ and $100 \mu \mathrm{l}$, respectively (Fig. 8).

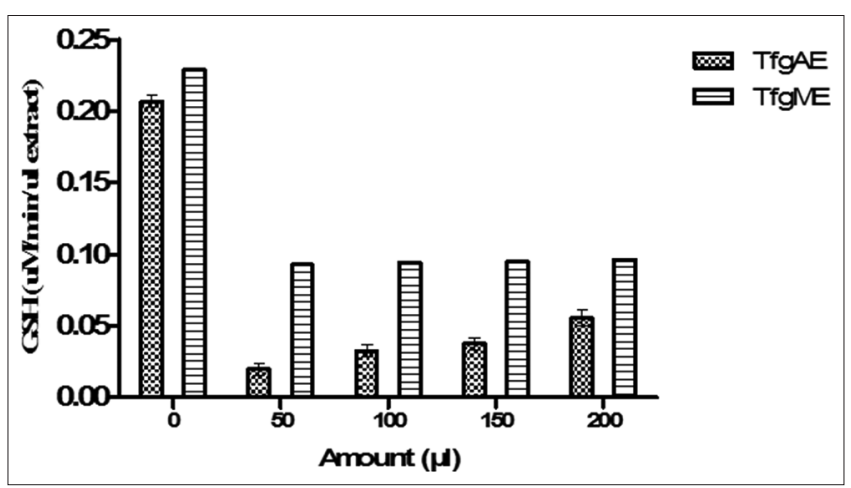

Fig. 5: Reduced glutathione (GSH) concentration in $\mu \mathrm{M}$ was estimated in the presence of methanolic and aqueous extract. The amount of reduced GSH increased with increase in the amount of extract. The experiment was performed in triplicates. Results were expressed in the form of means \pm standard error of mean.

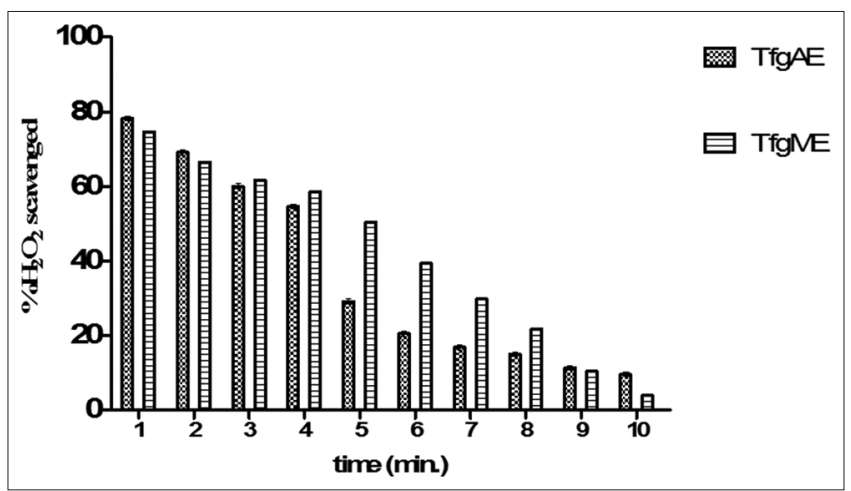

Fig. 6: Hydrogen peroxide scavenging (\%) was assessed with increasing amount of methanolic and aqueous extract. The hydrogen peroxide degradation increased in concentrationdependent manner. The experiment was performed in triplicates. Results were expressed in the form of means \pm standard error of mean.

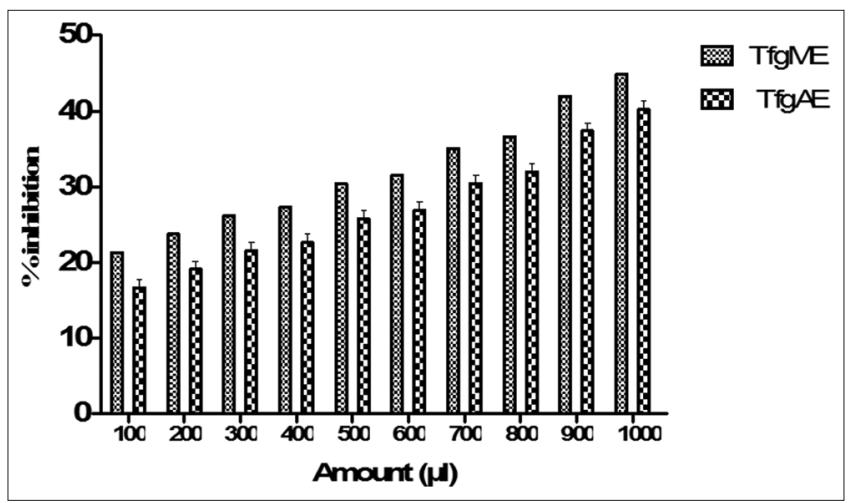

Fig. 7: Inhibition of albumin denaturation activity of a methanolic and aqueous extract of Trigonella foenum-graecum was checked at various concentrations taking aspirin $(100 \mu \mathrm{g} / \mathrm{ml})$ as standard.

The experiment was performed in triplicates. Results were expressed in the form of means \pm standard error of mean

\section{DISCUSSION}

T. foenum-graecum has been mostly used in ayurvedic medicines, and its seeds possess various kinds of bio-functional properties such as antimicrobial, anticholesterolemic, anti-carcinogenic, antiinflammatory, antiviral, antioxidant, hypotensive, laxative, uterine tonic, restorative, and galactogogue [10,39]. In addition, it provides several enzymatic properties, mitigates fever, reduces body pain and fat, augments appetite, alleviates swelling and induces lactation, and sex hormones. In the present study, the phytochemical analysis of extracts of powdered T. foenum-graecum seeds showed the presence of phenolic (tannins, total flavonoids, and phenols), and non-phenolics (alkaloids, sterol, resins, terpenoids, glycosides, saponins xanthoproteins, and quinines) phytocompounds while only carboxylic acid was not found. The medicinal importance of T. foenum-graecum plant was revealed by the presence of these secondary metabolites or bioactive compounds in the extracts. Furthermore, the methanolic extract gave a firm and excessive presence of bioactive compounds as compared to the aqueous extract. T. foenum-graecum plant depicts the therapeutic role in many diseases and does have various pharmacological properties that may be due to the presence of these bioactive compounds. Furthermore, the phytoconstituents which we have observed and reported here during the research work might be used for medicinal purpose. The presence of phytocompounds such as total phenols, flavonoids, and tannins may be responsible for nutraceutical and pharmacological activities such as anti-inflammatory, anti-oxidant antineoplastic, and antimicrobial [28-33].

Mostly plants possess potent antioxidant activity. Tannins and flavonoids are capable of scavenging free radical species and hence act as a classic antioxidant group in plants [34]. Free radicals are known to cause the aging of skin due to the failure action mechanism of natural antioxidants. Various in vivo and in vitro studies proved that the main cause of aging is the loss in the activity of enzymatic and non-enzymatic agents which enhance the level of reactive oxygen species (ROS) inside the cellular system. Ascorbic acid is one of the most important natural antioxidants as it oxidizes ascorbate to dehydroascorbate and therefore stamps out ROS. It may also be used to perform other several important physiological functions such as it might act as the cofactor for prolyl hydroxylase activity, which helps in the hydroxylation of prolyl and leads to the formation of procollagen and elastin $[35,40]$. It is common assumption that the cellular aging process can be prevented by plants' phenolic substances, which has galvanized the investigation of T. foenum-graecum metabolites and its possible, feasible action in the prevention and reduction of cellular aging. The result depicted the similar pattern of antioxidant activity by TfgAE, TfgME, and ascorbic acid (TfgME being higher than TfgAE) suggesting T. foenum-graecum as a potent antioxidant source.

Inflammation is just a common phenomenon after the reaction of living tissues toward injury. Some inflammatory vasodilator such as histamine, bradykinin, and prostaglandin- $\mathrm{E}_{2}$ acts as an effective vasodilator which elites the blood flow and redness in the region of acute inflammation [36]. Tannins are not only meant for their antiseptic property but also are important for their astringent action. This astringent property provides them the therapeutic value in arresting hemorrhage by constricting blood vessels and in protecting wounds, inflammation, and ulcer from external irritation by the participating surface protein which form an impervious protective coating on them. Thus, it is certain that the constituents are enough to cure the infection and tannins are also responsible to cure inflammatory diseases. The results obtained provide support for the use of $T$. foenum-graecum extract locally against inflammation, ROS related diseases, fever, ulcer, infection, etc. Alkaloids being bitter substance provide eminent antimicrobial action. Hence, it is immediate justifiable alkaloidcontaining plants that show effectiveness against the microbial diseases. The results show that TfgAE and TfgME both are having antibacterial activity in concentration-dependent manner which was sufficiently comparable to that of the standard drug. 


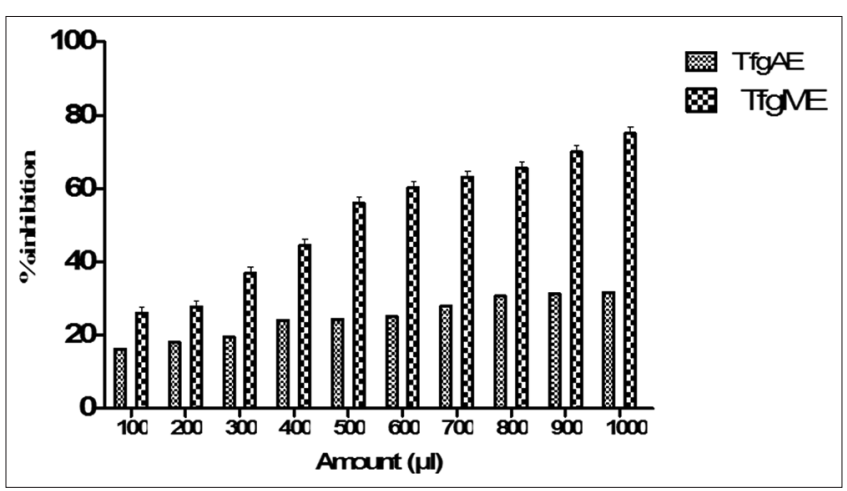

Fig. 8: Percentage inhibition of trypsin activity in the presence of a methanolic and aqueous extract of Trigonella foenum-graecum was evaluated. Experiment was performed in triplicates. Results were expressed in the form of means \pm standard error of mean.

The present work depicts homology with the previous study which validated that the constituents obtained from the seeds of T. foenumgraecum exhibited the potent antimicrobial activity. This may be due to the presence of competent bioactive compounds in the seed extracts of T. foenum-graecum which may be responsible for the destruction of the pathogenic microbes $[37,38]$. Finally, it can be concluded that these bioactive compounds can help in the treatment of bacterial infections. Results depicted from this study encouraging that this herb should be extensively studied and explored its utility in the prevention of several oxidative stressed, inflammatory diseases, etc.

\section{ACKNOWLEDGMENT}

The authors are thankful to the Department of Biotechnology, Jamia Millia Islamia, for providing the lab facility to carry out the research work.

\section{CONFLICTS OF INTEREST}

All the authors have no conflicts of interest. The manuscript has neither published nor simultaneously submitted for publication elsewhere. All the authors agree to the submission to the journal.

\section{REFERENCES}

1. Mullaicharam AR, Deori G, Uma-Maheswari R. Medicinal values of fenugreek-a review. Res J Pharm Biol Chem Sci 2013;4:1304-13.

2. Rababah TM, Ereifej KI, Esoh RB, Al-u'datt MH, Alrababah MA, Yang W. Antioxidant activities, total phenolics and HPLC analyses of the phenolic compounds of extracts from common Mediterranean plants. Nat Prod Res 2011;6:596-605.

3. Priya V, Jananie R, Vijayalakshmi K. GC/MS determination of bioactive components of Trigonella foenum graecum. J Chem Pharm Res 2011;5:35-40.

4. Gottfried SF. The raison of secondary plant substances. Science 2007; 129:1466-70.

5. Mehrafarin A, Rezazadeh SH, Naghdi BH, Noormohammadi GH, Zand E, Qaderi A. A review on biology, cultivation and biotechnology of fenugreek (Trigonella foenum-graecum L.) as a valuable medicinal plant and multipurpose. J Med Plants 2011;10:6-24

6. Betty R. The many healing virtues of fenugreek. India: Spice; 2008. p. 17-9.

7. Yoshikawa T, Toyokuni S, Yamamoto Y, Naito Y. Free Radicals in Chemistry Biology and Medicine. London: OICA International; 2000.

8. Miraldi E, Ferri S, Mostaghimi V. Botanical drugs and preparations in the traditional medicine of West Azerbaijan (Iran). J Ethnopharmacol 2001;2:77-87.

9. Basch E, Ulbricht C, Kuo G, Szapary P, Smith M. Therapeutic applications of fenugreek. Altern Med Rev 2003;8:20-7.

10. Kor NM, Moradi K. Physiological and pharmaceutical effects of fenugreek (Trigonella foenum-graecum L.) as a multipurpose and valuable medicinal plant. GlobJ Med Plant Res 2013;1:199-206.

11. Naidu MM, Shyamala B, Naik JP, Sulochanamma G, Srinivas P. Chemical composition and antioxidant activity of the husk and endosperm of fenugreek seeds. LWT Food Sci Technol 2011;2:451-6.

12. Rayyan S, Fossen T, Andersen OM. Flavone C-glycosides from seeds of fenugreek, Trigonella foenum-graecum L. J Agric Food Chem 2010;12:7211-7.

13. Belguith-Hadriche O, Bouaziz M, Jamoussi K, Simmonds MS, El Feki A, Makni-Ayedi F. Comparative study on hypocholesterolemic and antioxidant activities of various extracts of fenugreek seeds. Food Chem 2013;2:1448-53

14. Afef KE, Jan F, Alexander R, Siv T. Effects of dietary phenolic compounds on tocopherol, cholesterol and fatty acids in rats. Lipids 2000;35:427-35.

15. Raju J, Patlolla JM, Swamy MV, Rao CV. Diosgenin, a steroid saponin of Trigonella foenum graecum (Fenugreek), inhibits azoxymethaneinduced aberrant crypt foci formation in F344 rats and induces apoptosis in HT-29 human colon cancer cells. Cancer Epidemiol Biomarkers Prev 2004;8:1392-8.

16. Broca C, Manteghetti M, Gross R, Baissac Y, Jacob M, Petit P, Ribes G. 4-Hydroxyisoleucine: Effects of synthetic and natural analogues on insulin secretion. Eur J Pharmacol 2000;3:339-45.

17. Kaviarasan S, Naik G, Gangabhagirathi R, Anuradha C, Priyadarsini K. In vitro studies on antiradical and antioxidant activities of fenugreek (Trigonella foenum graecum) seeds. Food Chem 2007;1:31-7.

18. Harborne JB. Phytochemical Methods: A Guide to Modern Techniques of Plant Analysis. London: Chapman and Hall; 1998.

19. Barbade KD, Datar KG. Antibacterial activity, free radical scavenging potential, phytochemical investigation and in-vivo toxicity studies of medicinal plant Embelia basaal (R. \& S.) A. Dc. Asian J Pharm Clin Res 2015;8:171-7.

20. Goveas SW, Abraham A. Evaluation of antimicrobial and antioxidant activity of stem and leaf extracts of Coscinium fenestratum. Asian J Pharm Clin Res 2013;6:218-21.

21. Sudha G, Priya MS, Shree RI, Vadivukkarasi S. In vitro free radical scavenging activity of raw pepino fruit (Solanum muricatum aiton). Int J Curr Pharm Res 2011;3:137-40.

22. Kakkar P, Das B, Viswanathan PN. A modified spectrophotometric assay of superoxide dismutase. Indian J Biochem Biophys 1984;21:130-2.

23. Moron MS, Depierre JW, Mannervik B. Levels of glutathione, glutathione reductase and glutathione S-transferase activities in rat lung and liver. Biochim Biophys Acta 1979;582:67-78.

24. Jambunathan N. Determination and detection of reactive oxygen species (ROS), lipid peroxidation, and electrolyte leakage in plants. Methods Mol Biol 2010;639:292-8

25. Mizushima Y, Kobayashi M. Interaction of anti-inflammatory drugs with serum proteins, especially with some biologically active proteins. J Pharm Pharmacol 1968;20:169-73.

26. Oyedepo OO, Femurewa AJ. Anti-protease and membrane stabilizing activities of extracts of Fagra zanthoxiloides, Olax subscorpioides and Tetrapleura tetraptera. Int J Pharmacog 1995;33:65-9.

27. National Committee for Clinical and Laboratory Standards. Reference Method for Broth Dilution Antifungal Susceptibility Testing of Yeasts, Approved Standard M27-A3. Wayne, PA: National Committee for Clinical and Laboratory Standards; 2008. p. 14.

28. Lin Y, Shi R, Wang X, Shen HM. Luteolin, a flavonoid with potential for cancer prevention and therapy. Curr Cancer Drug Targets 2008;8:634-46.

29. Khacha-ananda S, Tragoolpua K, Chantawannakul P, Tragoolpua Y. Antioxidant and anti-cancer cell proliferation activity of propolis extracts from two extraction methods. Asian Pac J Cancer Prev 2013;14:6991-5

30. Pourmorad F, Hosseinimehr SJ, Shahabimajd N. Antioxidant activity, phenol and flavonoid contents of some selected Iranian medicinal plants Afr J Biotechnol 2006;5:1142-5.

31. Sengul M, Yildiz H, Gungor N, Cetin B, Eser Z, Ercisli S. Total phenolic content, antioxidant and antimicrobial activities of some medicinal plants. Pak J Pharm Sci 2009;22:102-6.

32. Hendra R, Ahmad S, Sukari A, Shukor MY, Oskoueian E. Flavonoid analyses and antimicrobial activity of various parts of Phaleria macrocarpa (Scheff.) Boerl fruit. Int J Mol Sci 2011:12:3422-31.

33. Rathee P, Chaudhary H, Rathee S, Rathee D, Kumar V, Kohli K. Mechanism of action of flavonoids as anti-inflammatory agents: A review. Inflamm Allergy Drug Targets 2009;8:229-35.

34. Polterait $\mathrm{O}$. Antioxidants and free-radical scavengers of natural origin. Curr Org Chem 1997;1:415-40.

35. Arunkumar K, Chandrashekar KR. Phytochemical evaluation and in vitro antimicrobial and antioxidant studies of leaf and stem bark extracts of Polyalthia fragrans (Dalz.) Bedd. An endemic species of 
western Ghats. Int J Pharm Pharm Sci 2017;9:20-4.

36. Masaki $\mathrm{H}$. Role of antioxidants in the skin: Anti-aging effects. J Dermatol Sci 2010;58:85-90.

37. Mohamed ST, Azeem AK, Dilip C, Sankar C, Prasanth NV, Duraisami R. Anti-inflammatory activity of the leaf extacts of Gendarussa vulgaris Nees. Asian Pac J Trop Biomed 2011;1:147-9.

38. Deans SG, Barrata MT. Antimicrobial and antioxidant properties of some essential oils. Flau Fragnance1998;13:235-44.
39. Nandagopal S, Dhanalakshmi DP, Kumar AG, Sujitha D. Phytochemical and antibacterial studies of fenugreek Trigonella foenum-graecum L.-A multipurpose medicinal plant. J Pharm Res 2012;5:413-5.

40. Widyawati PS, Werdani YD, Setiokusumo C, Kartikasari A. In vitro antioxidant capacities and antidiabetic properties of Pluchea leaves and green tea mixtures at various proportions. Int J Pharm Pharm Sci 2017;9:203-8. 